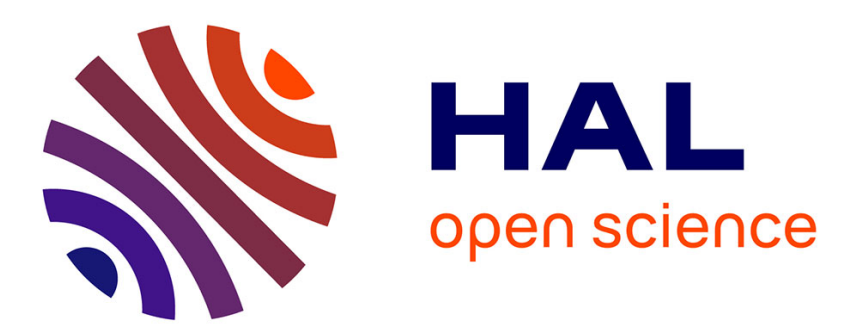

\title{
Photoinjection dans le fluorure de polyvinylidène
}

G. Guillaud, M. Maitrot

\section{To cite this version:}

G. Guillaud, M. Maitrot. Photoinjection dans le fluorure de polyvinylidène. Journal de Physique Lettres, 1982, 43 (15), pp.559-564. 10.1051/jphyslet:019820043015055900 . jpa-00232092

\section{HAL Id: jpa-00232092 https://hal.science/jpa-00232092}

Submitted on 1 Jan 1982

HAL is a multi-disciplinary open access archive for the deposit and dissemination of scientific research documents, whether they are published or not. The documents may come from teaching and research institutions in France or abroad, or from public or private research centers.
L'archive ouverte pluridisciplinaire HAL, est destinée au dépôt et à la diffusion de documents scientifiques de niveau recherche, publiés ou non, émanant des établissements d'enseignement et de recherche français ou étrangers, des laboratoires publics ou privés. 
Classification

Physics Abstracts

$61.40 \mathrm{~K}-72.20 \mathrm{~F}-72.40$

\title{
Photoinjection dans le fluorure de polyvinylidène
}

\author{
G. Guillaud et M. Maitrot \\ Laboratoire d'Electronique 2, Université Claude Bernard Lyon I, \\ 43, bd du 11 Novembre 1918, 69622 Villeurbanne Cedex, France
}

(Reçu le 10 mars 1982, révisé le 7 juin, accepté le 10 juin 1982)

\begin{abstract}
Résumé. - La photoconductivité du fluorure de polyvinylidène (PVDF) a été étudiée pour des longueurs d'onde inférieures à $3000 \AA$. Le courant est dû à des photoélectrons dont le parcours dans l'échantillon semble constant à partir d'une certaine tension. Un modèle de parcours constant dans un polymère ayant été proposé dans la littérature, une discussion est donnée.
\end{abstract}

\begin{abstract}
The photoconductivity of polyvinylidene samples is studied with $\lambda<3000 \AA$. Photoelectrons are injected and their range does not depend on voltage when this voltage is higher than a threshold value. A constant range model for carriers in a polymer was recently given in literature, and a discussion is given.
\end{abstract}

Introduction - Le fluorure de polyvinylidène (PVDF) est un matériau dont le comportement électrique a été très étudié ces dernières années [1]. Notamment, ce polymère présente des propriétés piézoélectriques et pyroélectriques qui sont maintenant exploitées dans des réalisations industrielles. Il a été montré en particulier [2] que l'effet piézoélectrique serait dû en partie à une injection de charges à partir de l'électrode positive bien que cela soit controversé.

Les mécanismes mis en jeu dans les phénomènes de conduction électrique : nature des porteurs, propriétés de transport, effets des électrodes sont encore mal connus. H. Sasabe et al. [3] ont étudié l'effet photovoltaïque dans PVDF, mais les résultats obtenus sur des échantillons $\alpha$ dopés ou $\beta$ à des longueurs d'onde supérieures à $3000 \AA$ sont au moins partiellement de nature pyroélectrique. Nous présentons ici des résultats concernant seulement la photoinjection dans des échantillons "purs » à des longueurs d'onde inférieures - en fonction du temps, de la tension appliquée, de l'intensité du flux lumineux et de la température. Des effets pyroélectriques existent dans nos échantillons dans le visible et l'infrarouge proche, mais feront l'objet d'un autre travail.

1. Dispositif expérimental - Les films de $100 \mu$ d'épaisseur ont été fournis par Kureha Chem. Soc. Leur configuration cristalline est essentiellement de la forme $\alpha$.

Des électrodes en or sont déposées sur chaque face de l'échantillon par évaporation sous vide. L'épaisseur des électrodes est contrôlée à partir de leur absorption optique et de leur résistance. L'échantillon est ensuite placé dans un four régulé qui peut être vidé $\left(10^{-6} \mathrm{~mm} \mathrm{Hg}\right)$.

La lumière incidente pénètre dans la cellule de mesure à travers une fenêtre en Spectrosil. La source lumineuse est une lampe à vapeur de xénon de $150 \mathrm{~W}$. Différents filtres interférentiels ou 
colorés sont utilisés pour rendre l'excitation lumineuse monochromatique. La variation de l'intensité du flux lumineux est obtenue à partir de filtres neutres.

Un problème important lors d'études des photocourants transitoires est l'élimination des courants parasites dus à la photoémission à partir des éléments métalliques de la cellule de mesure [4]. Il faut aussi disposer convenablement les sources de tension et les appareils de mesure dans le circuit électrique [5].

Un convertisseur courant-tension construit à partir d'un amplificateur opérationnel LF 356 associé à un oscillographe à mémoire Tektronix sont utilisés pour la mesure des photocourants.

Lorsque le polymère est éclairé par de la lumière non filtrée, on évite son échauffement en modulant par un disque tournant le faisceau incident [6]. La fréquence de modulation est de $55,5 \mathrm{~Hz}$; le rapport cyclique est de 6 , ce qui correspond à un temps d'éclairement de $3 \mathrm{~ms}$ par éclair.

2. Résultats expérimentaux. - On observe un photocourant seulement si l'électrode éclairée est négative. Les longueurs d'onde actives sont inférieures à $3000 \AA$ : des électrons sont ainsi injectés par l'électrode ou créés dans son voisinage par le faisceau incident.

La figure 1 montre la forme des courants transitoires obtenus avec une excitation monochromatique ( $2000 \AA)$ pour deux tensions de polarisation - l'échantillon étant illuminé à l'instant $t=0$ dès que le courant d'absorption dans l'obscurité est devenu négligeable.



Fig. 1. - Transitoires observés en éclairement monochromatique (2000 $\AA)$ à : (1) $100 \mathrm{~V}$ et (2) $200 \mathrm{~V}$. Epaisseur de l'échantillon $100 \mu$.

[Transients with monochromatic excitation $\lambda \sim 2000 \AA$. Applied voltage :(1) $100 \mathrm{~V}$ and (2) $200 \mathrm{~V}$. Sample thickness $100 \mu$.]

Cette réponse transitoire comprend deux parties : Dans un $1^{\mathrm{er}}$ temps le courant (après parfois une légère augmentation initiale) reste constant $\left(=i_{0}\right)$ pendant un certain temps. Il diminue ensuite brusquement, et dans un $2^{e}$ temps, il décroît lentement au cours du temps.

On obtient des courbes identiques lorsque la lumière est modulée, à condition de ne pas tenir compte du temps pendant lequel l'échantillon reste dans l'obscurité entre deux éclairs successifs (et tant que la fréquence de modulation n'est pas trop faible). On peut définir un temps $T_{1 / 2}$ au bout duquel le courant a décru de moitié $\left(i=i_{0} / 2\right)$.

Avec une tension suffisante $\left(V \geqslant 100 \mathrm{~V}\right.$, soit $\left.E \geqslant 10^{6} \mathrm{~V} / \mathrm{m}\right)$, la valeur de $i_{0}$ est indépendante de la tension appliquée. Elle est proportionnelle au flux lumineux incident (pour une répartition spectrale donnée). La longueur du palier de $i_{0}$ varie linéairement avec la tension et avec le flux lumineux. Le produit $i_{0} \times T_{1 / 2}$ représentant la charge $Q_{\mathrm{m}}$ mesurée dans le circuit extérieur jusqu'à l'instant $T_{1 / 2}$ est donc constant quel que soit le flux lumineux reçu. On a toujours $Q_{\mathrm{m}}=\alpha C V, C$ étant la capacité géométrique de l'échantillon, avec une valeur approximativement constante de $\alpha(\alpha<1)$. 


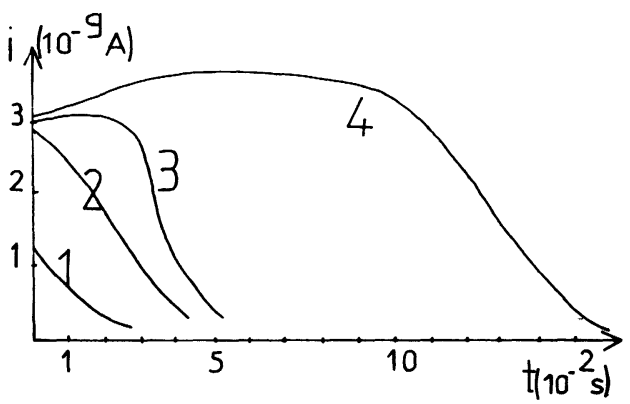

Fig. 2. - Transitoires observés en lumière totale modulée sous : (1) $2 \mathrm{~V}$, (2) $16 \mathrm{~V}$, (3) $30 \mathrm{~V}$, (4) $100 \mathrm{~V}$.

[Transients in total light with modulation. Applied voltage : (1) $2 \mathrm{~V}$, (2) $16 \mathrm{~V}$, (3) $30 \mathrm{~V}$, (4) $100 \mathrm{~V}$.]

$Q_{\mathrm{m}}$ a la même valeur quel que soit le mode d'éclairement de l'échantillon, c'est-à-dire sous illumination continue ou modulée. En fait, seul le temps total d'éclairement intervient.

A basse tension, le palier de $i_{0}$ n'est pas atteint, le courant décroissant rapidement avec le temps.

Si l'échantillon est court-circuité pendant un temps suffisant dans l'obscurité, l'expérience est reproductible. ( $\mathrm{Si}$ la mise en court-circuit est trop courte, on trouve $Q<Q_{\mathrm{m}}$ ). Le temps nécessaire à l'obtention d'une bonne reproductibilité est diminué si l'échantillon est court-circuité dans la lumière.

La valeur de $i_{0}$ ne dépend presque pas de la température, et on ne peut calculer une énergie d'activation, les très faibles variations obtenues peuvent être imputées à une non-reproductibilité due au vieillissement des échantillons.

Par contre la $2^{\mathbf{e}}$ partie du transitoire est activée (Fig. 3) et augmente avec la température. L'énergie d'activation mesurée après un temps $t=3 T_{1 / 2}$ est de l'ordre de $2 \mathrm{eV}$. Lorsque la température augmente, on atteint une température $T_{1}$ au-dessus de laquelle le courant dans l'échantillon devient constant. Si $T<T_{1}$, l'intensité $i_{2}$ de la $2^{\mathrm{e}}$ partie du transitoire est une fonction linéaire de $V$.

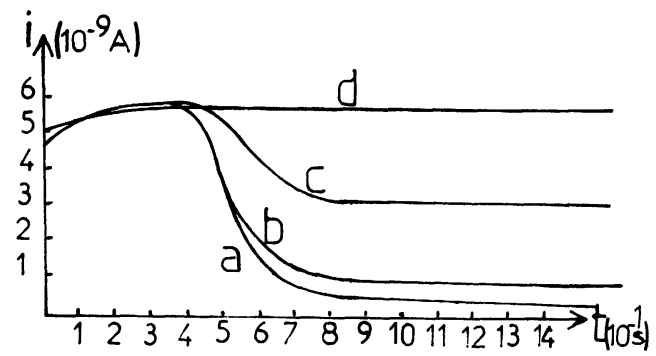

Fig. 3. - Transitoires observés en lumière totale modulée sous $300 \mathrm{~V}$ à différentes températures : a) $27^{\circ} \mathrm{C}$, b) $\left.\left.32^{\circ} \mathrm{C}, c\right) 52^{\circ} \mathrm{C}, d\right) 58^{\circ} \mathrm{C}$.

[Transients in total light $V=300 \mathrm{~V}$ for various temperatures :a) $\left.\left.27^{\circ} \mathrm{C}, b\right) 32^{\circ} \mathrm{C}, c\right) 52^{\circ} \mathrm{C}, d$ ) $58^{\circ} \mathrm{C}$.]

Il y a accroissement du courant au temps court (dépiégeage par la lumière de charges voisines de l'électrode).

3. Discussion. - Les résultats obtenus sont semblables à ceux que l'on a trouvés précédemment pour le nylon 66 [6]. Nous avions discuté de la photoconductivité obtenue dans ce cas en utilisant le modèle de Tabak et Scharfe [7]. 
- Comme dans le cas du nylon, en effet :

- Le courant initial est un courant injecté limité par l'électrode.

- On obtient deux « effets » successifs.

- La charge totale recueillie dans le circuit extérieur correspondant au $1^{\mathrm{er}}$ effet est de la forme $Q_{\mathrm{m}}=\alpha C V$.

Tabak et Scharfe ont proposé un modèle qui pourrait rendre compte des deux effets successifs observés : il y aurait transition d'une photoconductivité limitée par l'électrode à une photoconductivité limitée par la charge d'espace. En effet le piégeage des charges injectées crée une accumulation de charge d'espace dans l'échantillon. Quand la charge piégée atteint la valeur $C V$ le champ s'annule à l'électrode injectante et le courant devient limité par la charge d'espace. La transition entre les deux phénomènes a lieu pour $t \# T_{1 / 2}$.

A $t=T_{1 / 2}$ une charge $i_{0} T_{1 / 2}$ a été injectée dans l'échantillon. Si le temps de transit des charges est $L^{2} / \mu V$ et si $\tau$ est la durée de vie des électrons avant piégeage, le rapport du nombre de charges piégées au nombre de charges total ayant traversé l'échantillon est :

$$
\frac{L^{2}}{\mu V \tau} \quad\left(\operatorname{avec} L^{2} / \mu V \tau>1\right) .
$$

On a donc :

$$
i_{0} T_{1 / 2} \frac{L^{2}}{\mu V \tau}=\beta \varepsilon \frac{S V}{L}
$$

(avec $1<\beta<2$ et $S$ la surface de l'échantillon) de telle sorte que le produit $i_{0} T_{1 / 2}$ est proportionnel à $V^{2}$. $\lambda(V)$

Si au contraire $\tau<L^{2} / \mu V$ et si on néglige le dépiégeage on peut définir une longueur de parcours

$$
\lambda=\frac{\mu V \tau}{L}
$$

La charge mesurée $Q_{\mathrm{m}} \# Q_{\mathrm{t}} \frac{\lambda}{L}$, toutes les charges étant considérées comme piégées à la distance $\lambda$.

Dans ces conditions la charge piégée annulant le champ à l'électrode prend la forme [6] :

$$
\begin{aligned}
& Q_{\mathrm{t}}=\frac{C V}{\left(\frac{\lambda}{L}-1\right)} \\
& Q_{\mathrm{m}}=\frac{C V}{\left(\frac{\lambda}{L}-1\right)} \frac{\lambda}{L} \lambda \text { étant inférieur à } L .
\end{aligned}
$$

$Q_{\mathrm{m}}$ n'est pas linéaire avec $V$.

Les résultats expérimentaux montrent que $Q_{\mathrm{m}}$ est proportionnel à $V$ dans un grand intervalle de tensions. Il faudrait que le produit $\mu V \tau$ soit constant ( $\tau$ inversement proportionnel à $V$ ou $\mu V$ constant).

Or, une vitesse de déplacement des porteurs saturée avec le champ électrique a été récemment proposée dans le cas de polymères à une dimension [8].

Un tel modèle est peut être plus proche de la réalité pour un polymère ordonné comme PVDF. 
Dans le premier cas cependant, la charge mesurée dans le circuit extérieur devrait être supérieure à $C V$, ce qui est contraire aux résultats expérimentaux. Ceux-ci seraient mieux décrits en considérant le second cas, c'est-à-dire un parcours constant des porteurs, $\lambda=x_{0}$.

Ce parcours $x_{0}$ pourrait aussi être induit par la structure : longueur des chaînes ou inhomogénéités de l'échantillon (interfaces cristal-amorphe).

Il y a donc une différence fondamentale entre le modèle de Tabak et Scharfe (valable pour les amorphes minéraux) et les résultats obtenus dans le cas des polymères. $Q_{\mathrm{m}}=i_{0} T_{1 / 2}$ est proportionnel à $V^{2}$ dans le $1^{\text {er }}$ cas et à $V$ seulement dans le $2^{\mathrm{e}}$.

Dans le PVDF à température ordinaire les électrons ne traversent donc pas l'échantillon, et sont piégés à la distance $x_{0}$ - compte tenu des valeurs de $Q_{\mathrm{m}}$ et $\alpha$ on peut déduire $x_{0}$. Les mesures faites sur plusieurs échantillons ont donné des résultats un peu dispersés de $x_{0}$; la valeur moyenne de $x_{0}$ est environ de $10 \%$ de l'épaisseur du film (soit $10 \mu$ ). La $2^{\mathrm{e}}$ partie du transitoire a été étudiée en détail en lumière modulée à $30^{\circ} \mathrm{C}$. Sa variation avec la tension est très légèrement sous linéaire (Fig. 4). Or, si le modèle de Tabak et Scharfe s'appliquait au PVDF, ce courant limité par la charge d'espace devrait varier comme $V^{n}$ avec $n \geqslant 2$. Par contre si on suppose que la vitesse de déplacement des porteurs se sature avec le champ au-dessus d'une valeur $V_{\mathbf{s}}$ le courant doit varier comme $V$, ce qui est bien notre cas.

Ces courants semblent constants dans le temps. En réalité, ceci est dû à la modulation du faisceau lumineux comme le montre la figure 5 . Entre deux éclairs successifs il y a dépiégeage des charges.

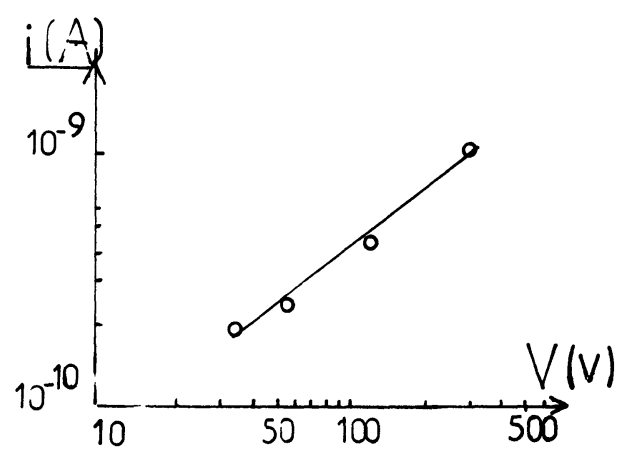

Fig. 4. - Intensité du « plateau » du second transitoire avec la tension : (température $30^{\circ} \mathrm{C}$ ).

[Intensity of the second transient versus voltage $\left(T=30^{\circ} \mathrm{C}\right)$.]

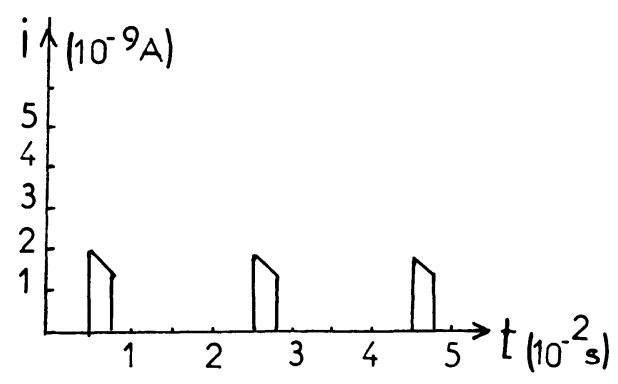

Fig. 5. - Représentation de 3 pulses de courant successives $(V=300 \mathrm{~V})$.

[Modulated light : three successive pulses $(V=300 \mathrm{~V})$.] 
A température élevée, le premier effet du transitoire est peu modifié. Ceci est normal dans la mesure où le parcours des porteurs est constant et où l'injection est limitée par l'électrode. Par contre, la $2^{\mathrm{e}}$ partie du transitoire est activée, soit par suite d'un dépiégeage, soit par suite d'une activation de la vitesse de déplacement des charges.

Enfin la mobilité des électrons injectés dans le PVDF doit être élevée et on peut donner une valeur limite au-dessous de laquelle il y aurait désaccord avec nos résultats : à $100 \mathrm{~V}$ par exemple le courant est nettement limité par l'électrode à̀ environ $6 \times 10^{-9} \mathrm{~A}$ pour une surface de $0,6 \mathrm{~cm}^{2}$ soit $10^{-8} \mathrm{~A} / \mathrm{cm}^{2}$ pour une épaisseur de $100 \mu$. Si le courant était limité par charge d'espace la mobilité correspondante serait dans le modèle classique environ $5 \times 10^{-3} \mathrm{~cm}^{2} / \mathrm{V}$.s. Comme nous sommes très loin de ce cas la mobilité doit être bien supérieure à cette valeur.

4. Conclusion. - La photoconductivité des échantillons de PVDF (fluorure de polyvinylidène) est, dans les conditions de notre expérience, limitée par l'électrode. Des électrons sont injectés dont le parcours avant piégeage semble constant à la température ordinaire.

La mobilité des charges mobiles est élevée pour un polymère, et supérieure à $5 \times 10^{-3} \mathrm{~cm}^{2} / \mathrm{V}$. $\mathrm{s}$.

\section{Bibliographie}

[1] Topics in Applied Physics, vol. 33, Sessler, G. M., Editor.

Block, H., Adv. Polym. Sci., vol. 33 (Springer International Editor).

[2] Latour, M., J. Physique-Lett. 41 (1980) L-35.

[3] Sasabe, H., Nakayama, T., Kumazawa, K., Miyata, S. et Fukada, E., Polym. J. 13 (1981) 967-973.

[4] Vermeulen, I. A., Wintle, H. J. et Nicodemo, D. A., J. Polym. Sci. A 2, 9 (1971) 543.

[5] Commins, J. D. et Wintle, H. J., J. Polym. Sci. 10 (1972) 2259.

[6] Guillaud, G., Rosenberg, N. et Maitrot, M., J. Polym. Sci. 18 (1980) 523.

[7] TabaK, M. D. et Scharfe, M. E., J. Appl. Phys. 41 (1970) 2114.

[8] Donovan, K. J. et Wilson, E. G., Philos. Mag. B 44 (1981) 31-45. 